\title{
Comparison of Particle Swarm and Simulated Annealing Algorithms for Induction Motor Fault Identification
}

\author{
S. A. Ethni, B. Zahawi, D. Giaouris and P. P. Acarnley \\ School of Electrical, Electronic \& Computer Engineering, Merz Court, Newcastle University, Newcastle upon Tyne NE1 7RU, \\ England, UK \\ Email: bashar.zahawi@ncl.ac.uk
}

\begin{abstract}
The performance of two stochastic search methods, particle swarm optimisation (PSO) and simulated annealing (SA), when used for fault identification of induction machine stator and rotor winding faults, is evaluated in this paper. The proposed condition monitoring technique uses time domain terminal data in conjunction with the optimization algorithm to indicate the presence of a fault and provide information about its nature and location. The technique is demonstrated using experimental data from a laboratory machine.
\end{abstract}

\section{INTRODUCTION}

The induction motor is without doubt the most widely used form of electric power device in any modern power system, giving power to millions of homes, farms and factories all over the world. The early detection of developing machine faults can therefore be vital if the costs of lost production arising from motor failures are to be avoided. Traditional induction machine condition monitoring techniques [1] usually involve the use of sensors embedded in the machine to measure, for example, temperature or vibration. There has also been considerable interest in detecting winding and other machine faults by examination of terminal current waveforms [2] using data gathered under steady-state operating conditions. Such techniques may involve the calculation of quantities such as input power [3] or negative sequence components [4]. Recent trends in condition monitoring include the detection of machine faults using data acquired during speed transients [5] and the estimation of machine parameters [6]. A comprehensive review of the current state of the art of induction machine diagnostic techniques, including the application of artificial intelligence techniques, is presented in [7].

A new technique for machine condition monitoring and fault identification using terminal and rotor position data had recently been proposed by the authors [8]. In this method, a stochastic search is carried out to estimate values of machine parameters which give the best possible match between the performance of the faulty experimental machine and its mathematical model, thus identifying both the location and nature of the winding fault. Fig. 1 shows a schematic diagram of the new fault identification technique. Stator currents are calculated from an $\mathrm{ABCabc}$ transient induction motor model and compared to the actual measured currents to produce a set of current errors that are integrated then summed to give an overall error function. When the machine is in its healthy state, there is a high correlation between its effective parameters and the model parameters resulting in a small calculation error. If a fault develops in the machine, its electrical parameters are of course modified and when the measured currents are compared with calculated currents there will be a large calculation error giving a fast indication that a fault of some type is present. Fault identification is carried out by adjusting the model parameters, using a stochastic search method to minimize the error. The new set of model parameters then defines the nature and location of the fault. Unlike many other methods, it should be noted here that the new stochastic search based approach does not require any expert prior knowledge of the type of fault or its location; both are identified as an integral part of the optimisation process.

In this paper, the application of two stochastic search algorithms, namely Particle Swarm Optimisation (PSO) and Simulated Annealing (SA) is investigated. The two algorithms are evaluated and their merits compared as alternative search techniques when dealing with the condition monitoring problem. The investigation is carried out using a laboratory three-phase, $240 \mathrm{~V}, 1.5 \mathrm{~kW}$ wound rotor induction machine.

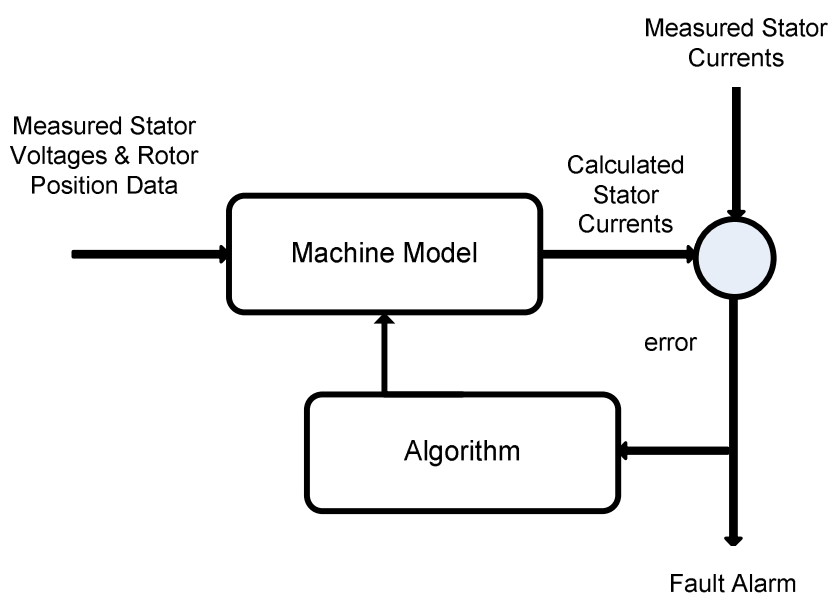

Fig. 1. Schematic representation of the stochastic search based fault identification technique. 


\section{PARTICLE SWARM OPTIMISATION}

Particle Swarm Optimisation is an iterative optimisation technique inspired by the biological behaviour of a swarm of birds or bees. Unlike evolutionary optimization techniques such as Genetic Algorithms, it is not based on the idea of the survival of the fittest. Instead, it is a collective method in which members of the population cooperate to find a global optimum in a partially random way and without any selection. Members of the population with the lower fitness functions are not discarded but do survive and can potentially be the future successful members of the swarm [9].

Each member of the population (or particle) $\mathbf{X}_{\mathrm{i}}$ is treated as a point in the $\mathrm{N}$-dimensional space representing the optimization problem, so that:

$\mathbf{X}_{\mathrm{i}}=\left(x_{\mathrm{i} 1}, x_{\mathrm{i} 2}, \ldots, x_{\mathrm{iN}}\right)$ for $\mathrm{i}=1,2, \ldots, \mathrm{M}$

where $\mathrm{M}$ is the number of particles that form the population and $\mathrm{N}$ is the number of variables.

The position of each particle within the search space is a potential result that can be evaluated in according to a given performance function to assess the fitness value of that particle. Each particle receives information from other members of the population about their best position to date and will also remember its own best position. The particle then calculates its next displacement vector in the search space (or velocity if we regard each step in the iterative process as representing a time unit of 1) as a combination of three factors: the particle's own velocity, moving towards the particle's own best position so far and moving towards the best position of its best informer, giving the following equations of motion for each particle:

$v_{n}^{k+1}=c_{1} v_{n}^{k}+c_{2}\left(p_{n}^{k}-x_{n}^{k}\right)+c_{3}\left(g_{n}^{k}-x_{n}^{k}\right)$

$x_{n}^{k+1}=x_{n}^{k}+v_{n}^{k+1}$

where $\mathrm{n}=(1,2, \ldots, \mathrm{N}), c_{1}$ is a constant representing the particle's confidence in its own movement, $c_{2}$ and $c_{3}$ are random numbers between 0 and $c_{\max }$ representing the weight the particle gives to its own previous best position and that of its informants, $p_{\mathrm{n}}$ is the position of the best informant and $g_{\mathrm{n}}$ is the particle's own best position.

If the search space is not infinite, it becomes necessary to confine the search space to prevent a particle leaving the search space all together. A simple mechanism for such confinement mechanism is described by the following operations [9]:

$$
x_{n}^{k} \notin\left[x_{\text {min }}, x_{\text {max }}\right] \Rightarrow\left\{\begin{array}{c}
v_{n}^{k}=0 \\
x_{n}^{k}<x_{\text {min }} \Rightarrow x_{n}^{k}=x_{\text {min }} \\
x_{n}^{k}>x_{\text {max }} \Rightarrow x_{n}^{k}=x_{\text {max }}
\end{array}\right.
$$

To apply this concept to condition monitoring of an induction machine or for machine parameter identification [10], each individual $\mathbf{X}_{\mathrm{i}}$ in the population represents one set of values of the machine parameters (say winding resistances
$R_{s A}, R_{s B}, R_{s C}, R_{r a}, R_{r b}$ and $R_{r c}$ ) where the resistance values must lie within a pre-defined search space.

\section{SIMULATED ANNEALING}

Simulated annealing is a stochastic search technique in which a randomly generated potential solution, $\mathrm{N}$, to a problem is compared to an existing solution, $\mathrm{O}$. The probability of $\mathrm{N}$ being accepted for investigation depends on the proximity of $\mathrm{N}$ to $\mathrm{O}$. If $\mathrm{N}$ is accepted, its suitability as a solution is evaluated according to a swap probability function and it may be chosen to replace O. Both the acceptance and swap probability functions depend on a temperature parameter $\mathrm{T}$, which reduces in value as the algorithm proceeds. In the context of our condition monitoring problem, the solutions $\mathrm{O}$ and $\mathrm{N}$, represent possible values of machine parameters (say the six winding resistances):

$$
\begin{gathered}
O=\left[R_{s A O}, R_{s B O}, R_{s C O}, R_{r a O}, R_{r b O}, R_{r c O}\right] \\
N=\left[R_{s A N}, R_{s B N}, R_{s C N}, R_{r a N}, R_{r b N}, R_{r c N}\right]
\end{gathered}
$$

where the parameters must lie within the allowed search space (Fig. 2).

$$
\begin{aligned}
& \begin{array}{cc}
\mathrm{X}_{\mathrm{ra}}, R_{r b}, R_{r c} & N=\left[R_{s A N}, R_{s B N}, R_{s C N}, R_{r a N}, R_{r b N}, R_{r c N}\right] \\
\mathrm{X}_{21} & \\
\vdots &
\end{array} \\
& \mathrm{X}_{11} \quad \mathrm{R}_{\mathrm{sA}}, R_{\mathrm{SB}}, R_{\mathrm{SC}} \quad \mathrm{X}_{12}
\end{aligned}
$$

Fig. 2. The organization of the machine parameters in the search space.

The acceptance probability of the new potential solution $P_{\mathrm{A}}$ is defined as:

$P_{A}=\exp \left(-\left(\frac{\text { displacement }}{r g}\right) *\left(\frac{T_{s}}{T}\right)\right)$

where $T_{\mathrm{s}}$, is the initial temperature value and $T$ is the current temperature value ( $T$ is high at the beginning of the process and reduces gradually every time a solution is replaced, mimicking the metal annealing process, hence the name simulated annealing).

For the six parameter problem outlined above, the range $r g$ is defined as:

$r g=\sqrt{\sum_{m=A, B, C, a, b, c}\left(R_{m \max }-R_{m \min }\right)^{2} / 6}$

The displacement is the distance between the previous solution $\mathrm{O}$ and the newly generated solution $\mathrm{N}$ :

displacement $=\sqrt{\sum_{m=A, B, C, a, b, c}\left(R_{m N}-R_{m o}\right)^{2} / 6}$

$P_{\mathrm{A}}$ is compared to a random value $r_{1}$ between $[0-1]$ to decide if $\mathrm{N}$ is to be accepted for further investigation. If $P_{\mathrm{A}}$ is smaller than $r_{1}, \mathrm{~N}$ is rejected and the process repeated. If $P_{\mathrm{A}}$ is greater than $r_{1}, \mathrm{~N}$ is accepted for further evaluation. At the 
beginning of the algorithm, when $T$ is high, $P_{\mathrm{A}}$ is high for all values of displacement meaning that there is a high probability that any new randomly generated potential solution is accepted for evaluation. This property is important to ensure that the entire search space is investigated and to prevent the algorithm converging to a local minimum. As the temperature reduces, $P_{\mathrm{A}}$ becomes small for large values of displacement concentrating the search on potential solutions that lie close to the existing solution.

The integral absolute error $E$ is then used to evaluate the swap probability function:

$$
P_{\text {swap }}=\frac{1}{1+\exp \left[\left(\frac{E_{N}-E_{O}}{E_{O}}\right) *\left(\frac{T_{s}}{T}\right)\right]}
$$

where $E_{\mathrm{N}}$ is the error obtained using the parameter set $\mathrm{N}$ and $E_{\mathrm{O}}$ is error obtained using the parameter set $\mathrm{O}$.

If $P_{\text {swap }}$ is smaller than a randomly generated number $r_{2}[0-$ 1], then the original set $\mathrm{O}$ is retained, and, if $P_{\text {swap }}$ is greater than $r_{2}$, the new set $\mathrm{N}$, is accepted and $\mathrm{O}$ is replaced by $\mathrm{N}$. Equation (7) shows that if $E_{\mathrm{N}}$ is smaller than $E_{\mathrm{O}}, P_{\text {swap }}$ is greater than 0.5 meaning that the better new parameter set is likely to be accepted. For lower temperatures, this probability approaches 1 . However, at the initial stages of the process when $T$ is high, there is a finite swap probability that a new worse solution is accepted. This feature of the algorithm helps prevent convergence to a local minimum.

In this investigation, the function proposed by Yao [11] is used to decrement the temperature function, with $T$ being inversely proportional to the number of successful potential solutions.

\section{MACHINE MATHEMATICAL MODEL}

Assuming that the machine has a smooth air-gap, the threephase machine voltage equation can be written in the natural $\mathrm{ABCabc}$ reference frame:

$\left[\begin{array}{c}v_{A} \\ v_{B} \\ v_{C} \\ v_{a} \\ v_{b} \\ v_{c}\end{array}\right]=\left[\begin{array}{cccccc}R_{A}+L_{s} p & M_{s} p & M_{s} p & M p \cos \theta_{1} & M p \cos \theta_{2} & M p \cos \theta_{3} \\ M_{s} p & R_{B} & M_{s} p & M p \cos \theta_{3} & M p \cos \theta_{1} & M p \cos \theta_{2} \\ M_{s} p & M_{s} p & R_{C} & M p \cos \theta_{2} & M p \cos \theta_{3} & M p \cos \theta_{1} \\ M p \cos \theta_{1} & M p \cos \theta_{3} & M p \cos \theta_{2} & R_{a}+L_{r} p & M_{r} p & M_{r} p \\ M p \cos \theta_{2} & M p \cos \theta_{1} & M p \cos \theta_{3} & M_{r} p & R_{b}+L_{r} p & M_{r} p \\ M p \cos \theta_{3} & M p \cos \theta_{2} & M p \cos \theta_{1} & M_{r} p & M_{r} p & R_{c}+L_{r} p\end{array}\right]\left[\begin{array}{c}i_{A} \\ i_{B} \\ i_{C} \\ i_{a} \\ i_{b} \\ i_{c}\end{array}\right]$

where $v_{\mathrm{A}}, v_{\mathrm{B}}, v_{\mathrm{C}}, i_{\mathrm{A}}, i_{\mathrm{B}}, i_{\mathrm{C}}$ are stator winding voltages and currents, $v a, v b, v c, i a, i_{\mathrm{b}}, i_{\mathrm{c}}$ are rotor winding voltages and currents, $R_{\mathrm{A}}, R_{\mathrm{B}}, R_{\mathrm{C}}$ are stator winding resistances, $L_{\mathrm{s}}$ is stator winding self inductance, $R_{\mathrm{a}}, R_{\mathrm{b}}, R_{\mathrm{c}}$ are rotor winding resistances, $L_{\mathrm{r}}$ is rotor winding self inductance, $M_{\mathrm{s}}$ is the mutual inductance between pairs of stator windings, $M_{\mathrm{r}}$ is the mutual inductance between pairs of rotor windings, $M$ is the peak value of rotor position dependent mutual inductance between stator/rotor winding pairs, $\theta_{1}$ is the rotor position angle (measured with respect to the A phase magnetic axis), $\theta_{2}=\theta_{1}+2 \pi / 3$ and $\theta_{3}=\theta_{1}+4 \pi / 3$.
Stator and rotor winding resistances are defined and subsequently adjusted during the search routine to minimise the current error function. Because the six winding resistances may have different values, there is no advantage in seeking to transform the machine equations into an alternative reference frame such as the DQdq reference frame. Instead the six winding voltage equations are simply subjected to the constraints imposed by winding connection (star or delta) and solved by numerical integration. Faults that will change the effective machine inductance values are not considered in this paper and will be included in future investigations.

\section{EXPERIMENTAL DATA}

A three-phase, $1.5 \mathrm{~kW}, 50 \mathrm{~Hz}, 240 \mathrm{~V}$, laboratory 2-pole wound-rotor induction motor was used to obtain experimental results for both faulted and healthy operating conditions. The machine had a star connected stator winding and a shortcircuited delta connected rotor winding. Standard tests (dc resistance, no-load and locked rotor tests) were carried out to determine the nominal values of the machine parameters, giving the following results:

$R_{\mathrm{A}}=R_{\mathrm{B}}=R_{\mathrm{C}}=5.88 \Omega, R_{\mathrm{a}}=R_{\mathrm{b}}=R_{\mathrm{c}}=6.83 \Omega, L_{\mathrm{s}}=0.729 \mathrm{H}$, $L_{\mathrm{r}}=0.578 \mathrm{H}, M_{\mathrm{s}}=0.25 \mathrm{H}, M_{\mathrm{r}}=0.70 \mathrm{H}$ and $M=0.769 \mathrm{H}$.

A developing open-circuit rotor winding fault was simulated by connecting a $7 \Omega$ resistor in series with the line connected to the two ends of the b-c rotor delta windings. This arrangement was used because it was not possible to gain access to the three separate delta connected windings. Data was collected over a time window of $0.2 \mathrm{sec}$, with a sampling interval of $1 \mathrm{~ms}$, with the machine operating at steady state with no load. The collected data set comprising the three stator currents is shown in Fig. 3.

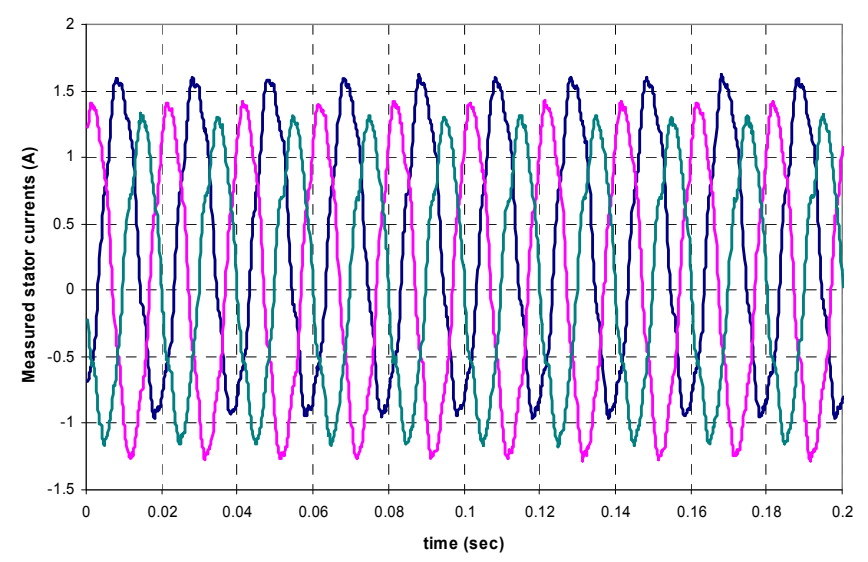

Fig. 3. Collected data set; stator currents.

The acquired data was then processed off-line using the PSO and SA algorithms to determine the effective resistances of the six windings. For the PSO algorithm, values of $c_{1}=0.5$ and $c_{\max }=1$ were used, together with a total swarm population of 24 particles and 3 informants per particle. For the SA algorithm the initial temperature is $T_{s}=10$. In both cases the error function was evaluated as:

$$
E=\sum\left(\left|i_{A m}-i_{A c}\right|+\left|i_{B m}-i_{B c}\right|+\left|i_{C m}-i_{C c}\right|\right) \Delta T
$$


where $\left(i_{\mathrm{Am}}, i_{\mathrm{Bm}}, i_{\mathrm{Cm}}\right)$ is the measured current set, $\left(i_{\mathrm{Ac}}, i_{\mathrm{Bc}}, i_{\mathrm{Cc}}\right)$ is the calculated current set and $\Delta \mathrm{T}$ is the sampling period.

\section{IDENTIFICATION OF ROTOR WINDING FAULT}

Figs. $4 \& 5$ show the values of stator and rotor resistances obtained by the PSO algorithm when used with the test data obtained from the faulted machine set. The error function produced by the solution is shown in Fig. 6. The number of steps or investigations required to obtain convergence of the two data sets was 29. The calculation error falls from a maximum value of 0.023 A.s, before reducing to 0.0167 As. Because of the simplicity of the machine model used in the investigation, it would be unrealistic to expect this error to reduce to zero, even with a much larger number of iterations. Clearly, the algorithm successfully detects the presence of the rotor winding fault as indicated by the high values of $R_{\mathrm{rb}}$ and $R_{\mathrm{rc}}$ in Fig. 5, the two windings connected to the external $7 \Omega$ resistor.

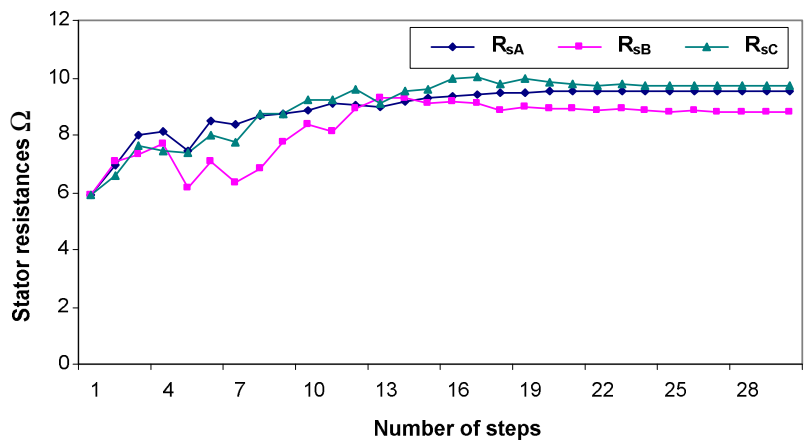

Fig. 4. Stator resistance estimation using PSO for operation with rotor winding fault.

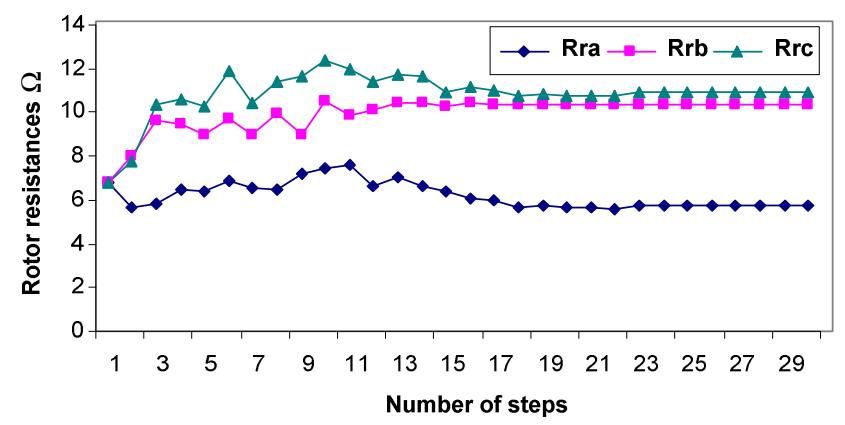

Fig. 5. Rotor resistance estimation using PSO for operation with rotor winding fault.

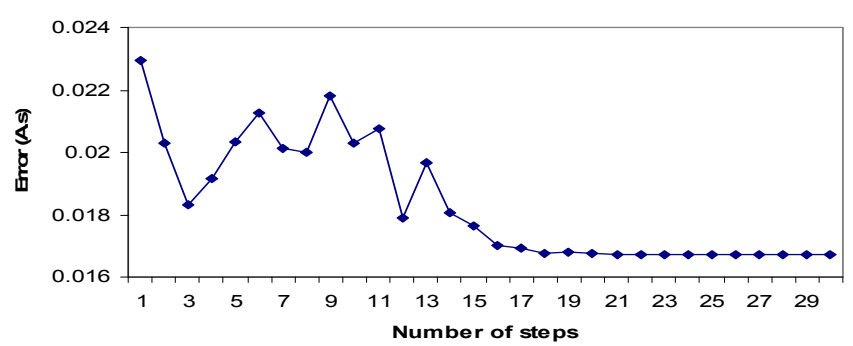

Fig. 6. Current estimation error using PSO for operation with rotor winding. fault
Figs. 7, $8 \& 9$ show the behaviour of the SA algorithm operating with the same faulted rotor data set. In this case, 46 successful investigations of potential solutions were required to obtain convergence with the calculation error falling from a maximum value of 0.0843 A.s to 0.0217 A.s.

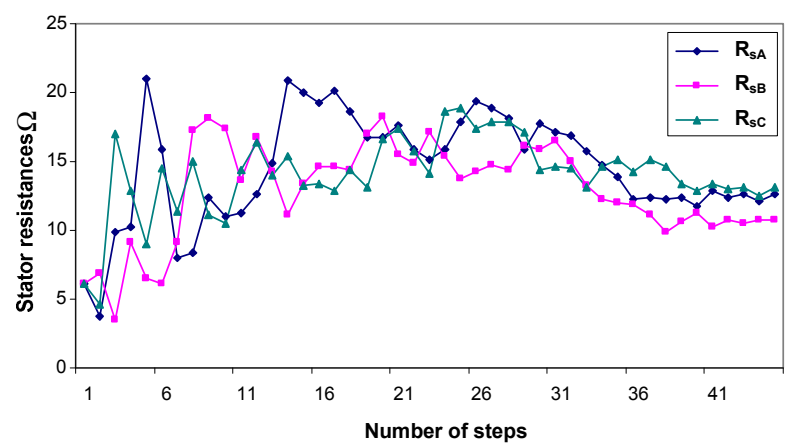

Fig. 7. Stator resistance estimation using SA for operation with rotor winding fault.

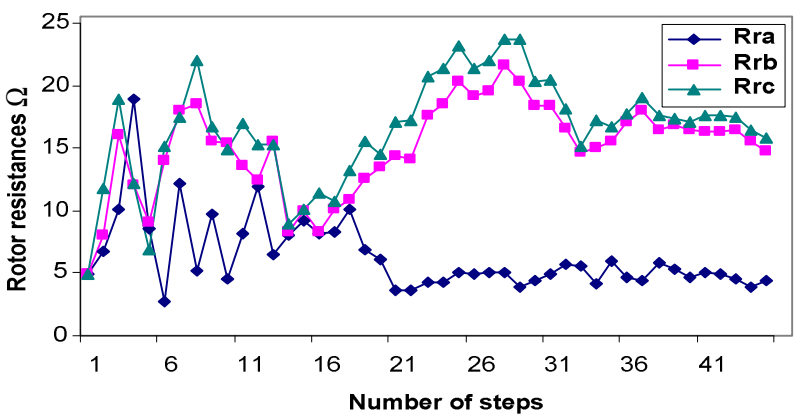

Fig. 8. Rotor resistance estimation using SA for operation with rotor winding fault.

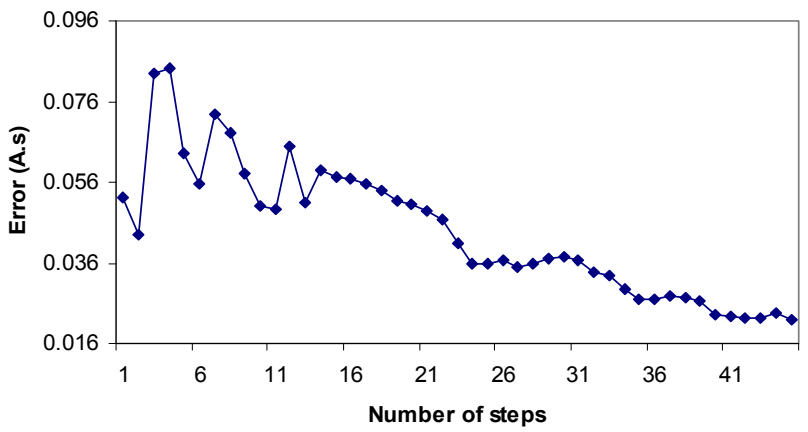

Fig. 9. Current estimation error using SA for operation with rotor winding fault.

The SA algorithm had a success rate of about $60 \%$ when used with the no-load measured current data compared with a success rate of about $99 \%$ for the PSO algorithm, which was also substantially faster than the SA algorithm which required a much larger number of investigations to produce consistent values for the estimated rotor resistances (the number of investigations when conducting a SA search being substantially larger than the number of accepted solutions). This demonstrates the robust nature of the PSO process and its suitability to this type of nonlinear multivariable 
optimization problem. Both algorithms showed estimated stator resistances to converge to similar values, confirming that there is no fault in the machine's stator windings.

\section{CONCLUSIONS}

The use of two stochastic search techniques (particle swarm optimisation and simulated annealing) to detect a developing induction motor winding fault has been presented. The condition monitoring method is based on the comparison of actual machine stator current data with that obtained from a simple mathematical model of the machine, and then using the stochastic search algorithm to minimise the resulting error function. Results show that the PSO algorithm is better suited for this type of application, achieving a success rate of about $99 \%$ compared with $60 \%$ for the SA algorithm with substantially improved execution times because of the smaller number of function evaluations needed for convergence.

Work is in progress to extend the number of machine parameters being estimated to include the inductances of the machine and thereby adopt a more rigorous approach to the identification of other machine faults, such as winding shortcircuit faults, machine mechanical faults and load faults.

\section{REFERENCES}

[1] P. J. Tavner and J. Penman, "Condition monitoring of electrical machines," Research Studies Press, Letchworth, England, 1987.

[2] M. E. H. Benbouzid, "A review of induction motors signature analysis as a medium for faults detection," IEEE Transactions on Industrial Electronics, vol. 47, no. 5, pp. 984-992, 2000.
[3] A. J. Trzynadlowski and E. Ritchie, "Comparative investigation of diagnostic media for induction motors: a case of rotor cage faults", IEEE Transactions on Industrial Electronics, vol. 47, no. 5, pp. 10921099, 2000.

[4] F. C. Trutt, J. Sottile and J. L. Kohler, "Online condition monitoring of induction motors," IEEE Transactions on Industry Applications, vol. 38 , no. 6 , pp. 1627-1632, 2002.

[5] H. Douglas, P. Pillay and A. K. Ziarani, "Broken rotor bar detection in induction machines with transient operating speeds," IEEE Transactions on Energy Conversion, vol. 20, no. 1, pp. 135-141, 2005.

[6] M. S. N. Said, M. E. H. Benbouzid and A. Benchaib, "Detection of broken bars in induction motors using an extended Kalman filter for rotor resistance sensorless estimation", IEEE Transactions on Energy Conversion, vol. 15, no. 1, pp. 66-70, 2000.

[7] A. Bellini, F. Filippetti, C. Tassoni and G. A. Capolino, "Advances in Diagnostic in Diagnostic Techniques for Induction Machines," IEEE Transactions on Industrial Electronics, vol. 55, no. 12, pp. 4109-4126, 2008.

[8] S. Ethny, P. P. Acarnley, B. Zahawi and D. Giaouris, "Induction Machine Fault Identification Using Particle Swarm Algorithms," PEDES 2006, IEEE International Conference on Power Electronics, Drives and Energy Systems for Industrial Growth, pp. 1-4, New Delhi, India, Dec 2006.

[9] M. Clerc, "Particle Swarm Optimizatio," ISTE Publishing Company, London, 2006.

[10] C. Picardi and N. Rogano, "Parameter Identification of Induction Motor Based on Particle Swarm Optimization", SPEEDAM 2006, International Symposium on Power Electronics, Electrical Drives, Automation and Motion, pp. 968-973, Taormina, Italy, May 2006.

[11] X. Yao, “A new simulated annealing algorithm”, International Journal of Computer Mathematics, vol. 56, pp. 161-168, 1995. 